\title{
Multiple tools for visualizing equipotential surfaces: Optimizing for instructional goals
}

\author{
Elizabeth Gire, ${ }^{1}$ Aaron Wangberg, ${ }^{2}$ and Robyn Wangberg ${ }^{3}$ \\ ${ }^{1}$ Department of Physics, Oregon State University, Corvallis, OR 97331 \\ ${ }^{2}$ Department of Mathematics, Winona State University, Winona, MN 55987 \\ ${ }^{3}$ Department of Physics, St. Mary's University of Minnesota, Winona, MN 55987
}

\begin{abstract}
Curriculum developers are interested in how to leverage various instructional tools - like whiteboards, Mathematica notebooks, and tangible models - to maximize learning. Instructional tools mediate student learning and different tools support learning differently. We are interested in understanding how the features of instructional tools influence student engagement during classroom activities and how to design activities to match tools with instructional goals. In this paper, we explore these questions by examining an instructional activity designed to help advanced undergraduate physics students understand and visualize the electrostatic potential. During the activity, students use three different tools: a whiteboard, a pre-programmed Mathematica notebook, and a 3D surface model of the electric potential. We discuss how the tools may be used to address the the instructional goals of the activity. We illustrate this discussion with examples from classroom video.
\end{abstract}

\section{RESEARCH QUESTIONS \& METHODS}

Different features of instructional tools support learning in different ways and to various degrees [1]. For example, current online homework systems are able to provide immediate feedback to students on their answers and individualized tutoring resources. However, they do not provide feedback on the details of student solutions and have difficulty handling the many forms student input, such as diagrams and paragraphs. Online homework systems support instructional goals related to students being able to solve short calculations and answer conceptual questions and are often used in large introductory physics courses. In contrast, with paper-based homework, students may provide detailed solutions that include algebra, diagrams, and text. Instructors can provided detailed feedback about the solution process, but this feedback is not immediate. Paper-based homework is used in more advanced physics courses where physics problems are longer and more complex and class sizes are smaller. In designing instruction, instructional tools should be selected carefully and used strategically to achieve the desired learning goals $[2,3]$.

In classroom activities, instructional tools might include whiteboards for students to draw on, laptop computers with relevant software, or other models/equipment. Classroom activities may include multiple tools or representations. Being able to understand and use multiple representations is an important learning goal in STEM [4]. In order to understand how an instructional tool supports learning, it is important to consider: how information is encoded with/in the tool; how learners access, transform, and share information with the tool; and what cognitive processing is needed when using the tool [2]. For example, many studies have compared the effectiveness of physical and virtual tools/laboratories. While some studies have shown that virtual tools can be equally or more effective than physical tools, other studies show that sometimes physical tools are more appropriate. A tool's effectiveness depends on the alignment of its affordances and constraints with the learning goals of the activity $[3,5]$.

Previous research in physics has compared the effect of different tools on students' conceptual understandings of physics ideas [5-7], while other research has examined how the different forms of symbolic tools effect how computations are performed [8]. The discussion in this paper focuses on the ways in which different tools used a classroom activity support student learning and engagement differently. Specifically, we discuss the tools used by groups of students in an activity about superposition of electrostatic potential. We consider the form of the tools, what information is represented with the tools, what transformations/manipulations students can do with the tools, and how many students can participate in using each tool. This study is part of a larger project to design activities for groups of students in advanced physics courses that leverage physical models and other representations effectively.

Our analysis is informed by observations of students doing the activity in class. Author EG used the activity in an upperdivision E\&M course with 13 groups of 3 students. Video recordings were made of 4 of these groups. One of the videos did not record audio and was excluded from the data set. Author RW also used the activity in an upper-division E\&M course with 8 students, but no video recordings were made of groups in her class. Each author viewed the classroom videos at least once and made notes. Author EG transcribed the videos and identified episodes that seemed to illustrate how they used the tools during the activity. The other authors reviewed these episodes and all authors discussed these episodes until a consensus interpretation was reached. This paper reports the most salient episodes.

\section{THE INSTRUCTIONAL ACTIVITY: DRAWING EQUIPOTENTIAL SURFACES}

\section{A. Instructional goals}

The primary goals of the instructional activity are for students to be able to:

Goal 1 Superposition: find an electric potential field by adding the potential from each discrete charge. 
Goal 2 3D Function: explain that electrostatic potential is a function of three spatial variables and equipotential surfaces are surfaces in 3D space.

Goal 3 Contour Plot: create a contour plot of potential due to multiple discrete sources; explain where contour lines should be more or less dense; explain shape of contours very close/far from point charges.

Goal 4 Graphical Representations of Potential: interpret and compare various representations of a 3D scalar field and 2D slices of the field.

Goal 5 Inquiry: investigate physical phenomena.

\section{B. Structure of the instructional activity}

During the activity, groups of 2-3 students were given a large (2'x3') whiteboard with 4 dots arranged in a square. The students were told these dots represented 4 positive charges and were asked to draw equipotential surfaces of the configuration. After they produced a drawing on the whiteboard, the instructor led a whole class discussion about strategies for drawing the surfaces. The intent was for the students to reason about the equation of the potential for each point charge - how the function varies with distance and how the functions add together - in order to create this drawing.

Students were then provided with a Mathematica notebook pre-programmed with five different ways to visualize equipotential surfaces for five different distributions of point charges, including four positive charges arranged in a square. The intent was for the students to be made aware of various approaches to dealing with the difficulty of visualizing 3D scalar functions and for students to leverage their understanding of the contour plot that they produced to understand the other representations. The ways of visualizing the potential (Fig. 1) in Mathematica include:

1. a 3D set of cross-sections parallel to the plane of charges, using color to represent the potential;

2. a $2 \mathrm{D}$ contour plot of one cross-section;

3. a $3 \mathrm{D}$ plot of the potential in the plane of the charges (or a parallel plane) using the 3rd axis to represent the value of the potential;

4. a movie showing the potential in planes parallel to the plane of the charges, using color to represent the value of the potential; and

5. a 3D contour plot of the potential function.

While demonstrating the Mathematica notebook for the students, the instructor led a discussion about the connection between the ways of visualizing potential in Mathematica.

The students were then asked to consider a quadrupole (two positive and two negative charges, with like charges on opposite corners of the square) and to draw equipotential surfaces on their whiteboards. During the discussion, students
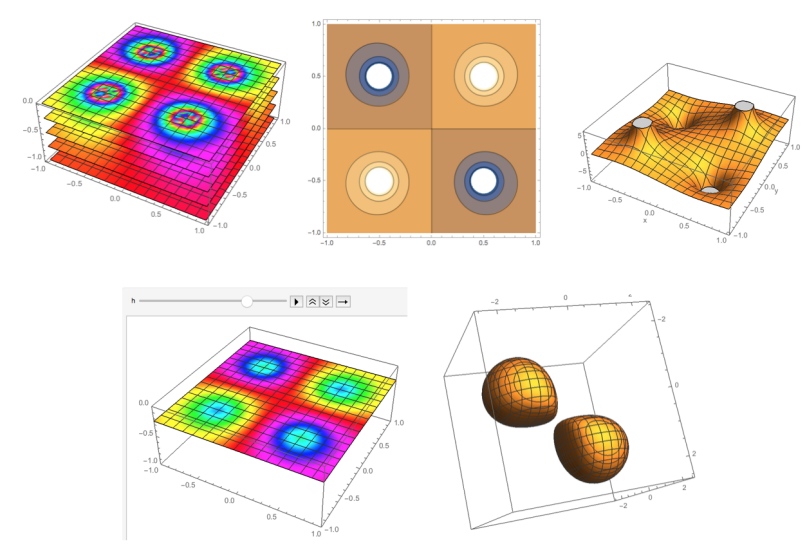

FIG. 1. Visualizations included in Mathematica worksheet evaluated for a quadrupole: 3D set of cross-sections (top left), 2D contour plot of one cross-section (top center), 3D surface plot of the potential function for a plane (top right), a frame of a movie of cross-sections (bottom left) and a 3D contour plot (bottom right).
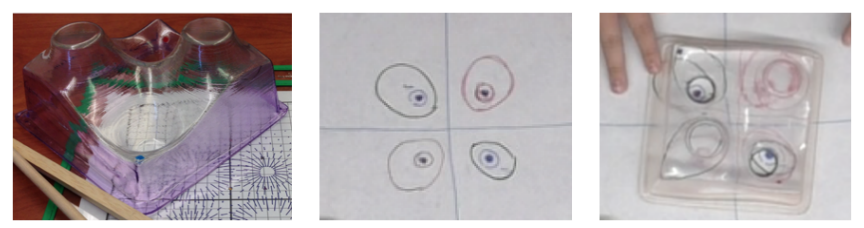

FIG. 2. Surface model of the potential due to a quadrupole in the plane of the charges (left), whiteboard contour map (center), and contours drawn on surface model and placed on whiteboard contour map (right).

were given surface models representing the potential in the plane of the charges. The surfaces use height to represent the value of the potential, similar to the surface plot produced by Mathematica (Fig. 1, top right). The models have a base of 6.5" 6.5 ", a height up to 5.5" and are transparent and dryerasable (Fig. 2, left) [9]. The instructional activity ended with a whole class discussion, incorporating student ideas and leading to statements of the main instructional goals.

\section{ANALYSIS}

\section{A. Making decisions vs. explanations}

Tools mediate learning by changing the nature of the instructional task. When using the whiteboard, all information that was added to the tool was added by the students, and we observed students making many decisions about how to draw the contours. Students generally started by considering the equation of the electric potential due to a point charge and some limiting cases: what the equipotential surfaces look like very far from the charge distribution (a circle with $\mathrm{V}=0$ ) and very close to the point charge (circles with a large positive or negative value of the potential). Then students typically 
used the equation to identify other locations where the potential might be zero and to estimated the potential at special locations like the midpoints between charges.

In contrast, the Mathematica notebook and the surface model does the superposition for the students. We observed that students used these tools in a looking-up-the-answer mode. When the Mathematica plots were different from the students' drawings or if the students did not feel confident in guessing a shape for the drawing, we observed that students tried to use physical reasoning to explain the correct Mathematica plot or contours determined with the surface model.

For example, one group became stuck while discussing how to determine the space of the equipotential curves inside the square. One of the students suggests using Mathematica to find out.

Anna: Yeah, so is this distance supposed to be bigger, or is this distance supposed to be bigger? That's what I'm wondering. (pause) Can we try it over there? (points to the laptop)

Charlie: Yeah, why try to visualize when the computer can do the work for us?

[some discussion about manipulating the code to make the charge distribution a quadrupole]

Anna: Does that? That looks right.

Charlie: Oh, hey. That's exactly what it is.

Bailey: [chuckles] That's funny. All right. So let's just think of this picture then. [Anna evaluates a new Mathematica cell] What?

Charlie: Yeah, I was right! On the asymptotes it's zero because along those lines, there's equal push and pull.

Anna: Right. And then, yeah, so it is actually spaced farther out that way and closer this way [points to the computer screen]. So it's the opposite of what you drew. [starts altering whiteboard drawing]

Bailey: So let's think about why.

In this episode, we see that these students developed questions about the spacing of the contours, used the Mathematica notebook to find out what the contours are supposed to look like, and then began a process of trying to explain why the contours looked the way that they did. We also see Charlie was excited that the Mathematica visualization supported his idea that the symmetry lines (what he called asymptotes) have zero potential, and he described a physical reason for this result, albeit an incorrect one. We also find it interesting and encouraging that, in this case, the tools helped the students overcome their stuckness without an intervention from an instructor.

Therefore, we see evidence that the use of the whiteboard supported Goals 1, 3 \& 5 (operationalizing superposition, understanding contour maps, and inquiry) by providing opportunities for decision-making that led to generating questions. The Mathematica visualizations and the surface model provided additional support when the students became stuck with their whiteboard drawings. The information provided by these tools acted as a foothold for further physical reasoning.

\section{B. 3D visualization of potential}

Mathematica offered several ways of plotting functions that allowed students to visualize the 3D nature of the field, using either the ability to rotate 3D plots or using time to cycle through cross-sections (Fig. 1). One limitation of these representations was that, although they can be rotated on the screen, interpreting 3D representations on a 2D screen was sometimes difficult for students.

In contrast, the tangible surface model was easier for some students to interpret. However, the surface model only represents the potential in a single plane.

The 2D nature of the whiteboard best supports 2D representations, like a contour plot of the potential in a plane. However, we saw that some students found surface plots more intuitive than contour plots. Before students were given the surface models, one student attempted a $3 \mathrm{D}$ perspective drawing of the surface. (Fig. 3).

Ethan: So, the only way I can actually, I can't do this [the contour plot] visually. I go to this [surface perspective drawing].
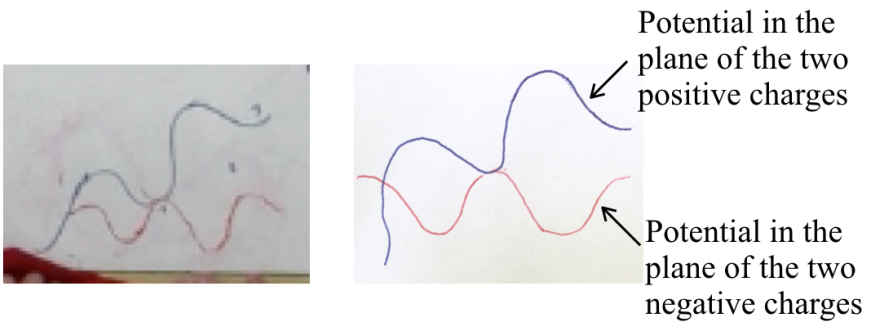

FIG. 3. Ethan's perspective drawing of the potential surface in the plane of the charges (right) and a high resolution reinterpretation of Ethan's drawing (left).

Therefore, we see that the Mathematica notebook best supported visualizing the potential as a 3D function (Goal 2).

\section{Co-locating representations for making connections}

The Mathematica representations included a digital version of the surface plot and the contour map (Fig 1, top right and center). These plots must be view side-by-side on the screen. With the surface model, however, we observed students drawing equipotential contours directly onto the surface model and place model over the contour map drawn on the whiteboard (Fig 2, right and center, and Fig. 4, bottom). The surface 

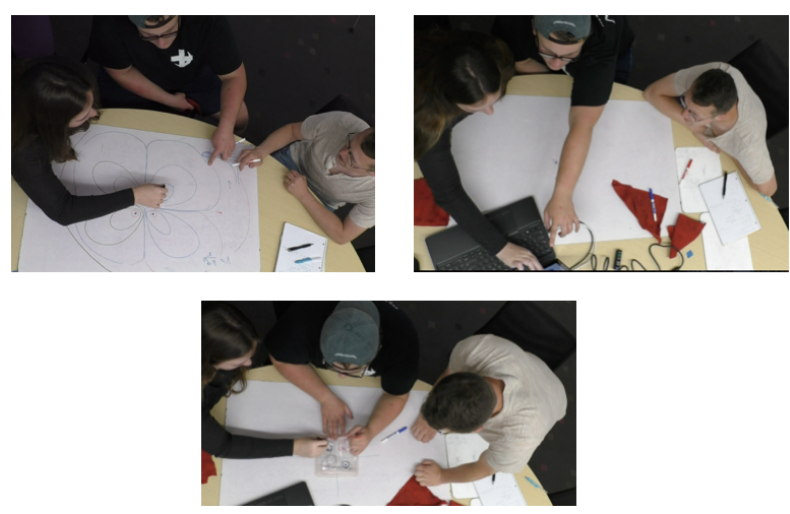

FIG. 4. Students using the instructional tools: three students writing on or pointing at whiteboard (top left), two students pointing to Mathematica notebook on a laptop screen (top right), and two student drawing on surface model while one student views contours from above (bottom).

model is transparent and allowed students to perceive the connection between the spacing of the level curves and the slope of the surface directly (Goals $3 \& 4$ ).

\section{Group manipulation of the tools}

The different tools were differently accessible to the students within the groups. Each student had similar access to the whiteboard. The whiteboard was centrally located and each student had a pen and an eraser. Usually one student did most of the writing/drawing, but we observed occasions where multiple members of the group wrote simultaneously (Fig. 4).

Similarly, the surface model is large enough for all students to access and draw on it during discussions. It is lightweight and transparent and we observed students place it centrally on top of the whiteboard.

Access to the Mathematica notebook was more limited. Typically, only one member of the group operated the laptop. Students tended to keep the laptop to one side of the whiteboard and occasionally some students were not able to see the monitor easily (Fig. 4, top right). However, the students who could see the monitor sometimes made suggestions to the laptop operator and interpreted plots. Therefore, we find that the whiteboard and the surface supported interaction from all group members (Goal 5) more than the Mathematica notebook.

\section{DISCUSSION}

We have discussed how the different features of three instructional tools - a whiteboard, a Mathematica notebook, and a surface model - mediated student learning during an instructional activity. The whiteboard allowed for students to reason about a superposition of fields in order to make decisions about how to draw the contour lines. We discussed an example where this decision making process led to the students generating productive questions. In contrast, Mathematica and the surface perform the superposition for the student, and can provide support when students reason incorrectly or when they get stuck. Combinations of tools like this - one that leads students to ask questions and one that provides information that helps students address those questions - are desirable for supporting students in inquiry processes.

Tools that could be centrally located in the group better supported engagement for all students in the group. This is important for three reasons. First, when each student participates, more ideas may be brought to bear to the discussion and the discussion may be richer. Second, having equal access to tools might reduce the marginalization of students within groups. Third, if students are expected to learn certain skills or ways of reasoning during the activity, each students ought to have opportunity to practice those skills.

Mathematica has various options for visualizing the 3D nature of the field, but the surface model allows the surface plot and the contour maps to be co-located. This co-location highlights the relationship between these representations.

In this paper, we discuss the role of tools during one instructional activity in one classroom. Our analysis informs our understanding of this particular activity and how these tools might be used effectively in other instructional contexts. However, we are cautious in making generalized claims from these data alone. In future work, we would like to examine more closely how the features of the tools promote or suppress student inquiry and collaboration.

\section{ACKNOWLEDGMENTS}

The Paradigms in Physics project (DUE-1323800) and the Raising Physics to the Surface project (DUE-1612480) are supported by the NSF.
[1] E. Hutchins, Cognition in the Wild, A Bradford book (MIT Press, 1995).

[2] S. Ainsworth, Learning and instruction 16, 183 (2006).

[3] T. de Jong, M. C. Linn, and Z. C. Zacharia, Science 340, 305 (2013).

[4] A. V. Heuvelen, American Journal of Physics 59, 891 (1991).

[5] Z. Chen and G. Gladding, Phys. Rev. ST Phys. Educ. Res. 10, 010111 (2014).

[6] J. J. Chini, A. Madsen, E. Gire, N. S. Rebello, and S. Puntam- bekar, Phys. Rev. ST Phys. Educ. Res. 8, 010113 (2012).

[7] N. S. Podolefsky and N. D. Finkelstein, Phys. Rev. ST Phys. Educ. Res. 3, 020104 (2007).

[8] E. Gire and E. Price, Phys. Rev. ST Phys. Educ. Res. 10, 020112 (2014).

[9] Additional information about the surface models at: http://www.raisingstem.org. 